\title{
DOS ESPECIES NUEVAS DE VALERIANA (VALERIANACEAE) DEL CENTRO DE MÉXICO ${ }^{1}$
}

\author{
JERZY RZEDOWSKI \\ Y \\ Graciela Calderón de Rzedowski \\ Instituto de Ecología, A.C. \\ Centro Regional del Bajío \\ Apartado postal 386 \\ Pátzcuaro, Michoacán
}

\begin{abstract}
RESUMEN
Se describen como nuevas dos especies de Valeriana, de distribución aparentemente muy restringida. $V$. emmanuelii, que procede de dos localidades ubicadas en el extremo sur del municipio de Morelia, Michoacán, es cercana a $V$. retrorsa Fern. y a $V$. densiflora Benth. Del estado de Querétaro se reconoce a $V$. zamoranensis, perteneciente a la serie Ceratophyllae, que sólo se registra de la parte más alta del Cerro Zamorano; al parecer está relacionada con $V$. zapotecana Barrie y con $V$. otomiana Barrie.
\end{abstract}

Palabras clave: México, Valeriana, Valerianaceae.

\section{ABSTRACT}

Two species of Valeriana of apparently very restricted distribution are described as new. $V$. emmanuelii, collected in two localities of the southern extreme of the municipality of Morelia, Michoacán, is related to $V$. retrorsa Fern. and to $V$. densiflora Benth. $V$. zamoranensis belongs to the series Ceratophyllae and is only known from the highest part of Cerro Zamorano; it seems to be related to $V$. zapotecana Barrie and to $V$. otomiana Barrie.

Keys words: Mexico, Valeriana, Valerianaceae.

La familia Valerianaceae está representada en México solamente por el género Valeriana. El estudio de sus representantes encaminado hacia su integración a la Flora del Bajío y de regiones adyacentes ha revelado la existencia de materiales de herbario que no corresponden a ninguna especie conocida de este grupo. El propósito de la

${ }_{1}^{1}$ Trabajo realizado con apoyo económico del Instituto de Ecología, A.C. (cuenta 902-03), del Consejo Nacional de Ciencia y Tecnología y de la Comisión Nacional para el Conocimiento y Uso de la Biodiversidad. 
Acta Botanica Mexicana (2003), 62: 65-71

presente contribución consiste en describir dos nuevos taxa encontrados, ambos aparentemente de distribución muy restringida, una del estado de Michoacán y una de Querétaro.

Valeriana emmanuelii Rzedowski \& Calderón sp. n.

Herba perennis ut videtur hermaphrodita $20-40 \mathrm{~cm}$ alta; radix napiformis vel fusiformis usque $7 \mathrm{~cm}$ longa et $2 \mathrm{~cm}$ lata, ramosa et gemmifera; caulis solitarius vel plures; folia plerumque longe petiolata maximam partem basalia, caulina in 1-2(3) paribus, laminae interdum simplices cordiformes vel reniformes ad $2 \mathrm{~cm}$ longae et $2.5 \mathrm{~cm}$ latae, saepe trifoliolatae vel aliquanto 2-4(5)-jugatae, foliolum terminale plerumque laminae simplicis simile, quam lateralibus valde majus; inflorescentia dichasiali-paniculata laxa, 3.5-5 cm longa et 4-6 cm lata; corolla alba hypocraterimorpha ca. $4.5 \mathrm{~mm}$ longa, extus glabra, intus pilis erectis paucis; stamina stylusque exserta; fructus ovatus vel pyriformis ca. $2.2 \mathrm{~mm}$ longus utrinque dense puberulus, pagina abaxialis 3-nervata, 2 nervos laterales incrassatos ad margines parallelos ferens.

Planta herbácea perenne, aparentemente hermafrodita, de 20 a $35(40) \mathrm{cm}$ de alto, a menudo formando conjuntos gregarios, subglabra, con los nudos del tallo y de la inflorescencia pubescentes; raíz manifiestamente engrosada, napiforme o fusiforme, hasta de $7 \mathrm{~cm}$ de largo por 1 a $2 \mathrm{~cm}$ de diámetro, frecuentemente ramificada, gemífera, propiciando la reproducción vegetativa; tallo uno o varios, sin ramificar, verde o a veces tendiendo a morado, sobre todo hacia la parte superior, de ca. $1 \mathrm{~mm}$ de diámetro, dando origen a una inflorescencia terminal; hojas por lo general numerosas y mayormente agrupadas en forma de roseta basal densa, con 1 ó 2 (3) pares de hojas caulinas, peciolo delgado, en las hojas basales de 5 a 10(12) cm de largo, en las caulinas mucho más reducido, láminas simples a veces presentes, acorazonadas a reniformes, de 1.5 a $2 \mathrm{~cm}$ de largo por (1.5)2 a $2.5 \mathrm{~cm}$ de ancho, de ápice obtuso a redondeado, base truncada a cordada, margen entero a ligeramente crenado, con más frecuencia las láminas son trifolioladas o pinnadas con 2 a 4(5) pares de foliolos laterales, siendo entonces oblongas en contorno general, hasta de $10 \mathrm{~cm}$ de largo por 4 a $5 \mathrm{~cm}$ de ancho, con el foliolo terminal con frecuencia semejante en forma y tamaño a la lámina simple (aun cuando a veces tiende a ser triangular o más a menudo circular y medir hasta $3 \mathrm{~cm}$ de diámetro, con el borde entero o subentero a irregularmente crenado), foliolos laterales sésiles, mucho más pequeños que el terminal, subcirculares a oblongos o lanceolados, hasta de $1.5(2) \mathrm{cm}$ de largo, pero con frecuencia los inferiores diminutos, hojas caulinas por lo común con menor número de foliolos laterales, todas glabras y de textura membranácea; inflorescencia terminal, inicialmente densa y capituliforme, más abierta en la madurez, de 3.5 a $5 \mathrm{~cm}$ de largo y de 4 a $6 \mathrm{~cm}$ de ancho, en forma de panícula dicasial, con las ramas ascendentes, ramillas terminales por lo general con 2 ó 3 flores, brácteas lineares a lanceoladas, de 2 a 5(6) mm de largo; flores al parecer todas hermafroditas; cáliz de (8)10 a 13 segmentos de alrededor de $3.5 \mathrm{~mm}$ de largo al expanderse; corolas blancas, hipocraterimorfas, con el tubo giboso en su base, de ca. $3 \mathrm{~mm}$ de largo y $1 \mathrm{~mm}$ de diámetro, los lóbulos patentes, oblongos, de ca. $1.5 \mathrm{~mm}$ de largo, redondeados en el ápice, glabras por fuera, por dentro provistas en el tubo de escasos pelos erectos de cerca de $1 \mathrm{~mm}$ de largo; estambres largamente exsertos, anteras de ca. $0.8 \mathrm{~mm}$ de largo; estilo 
Rzedowski y G. Calderón de Rzedowski: Dos Especies Nuevas de Valeriana de México

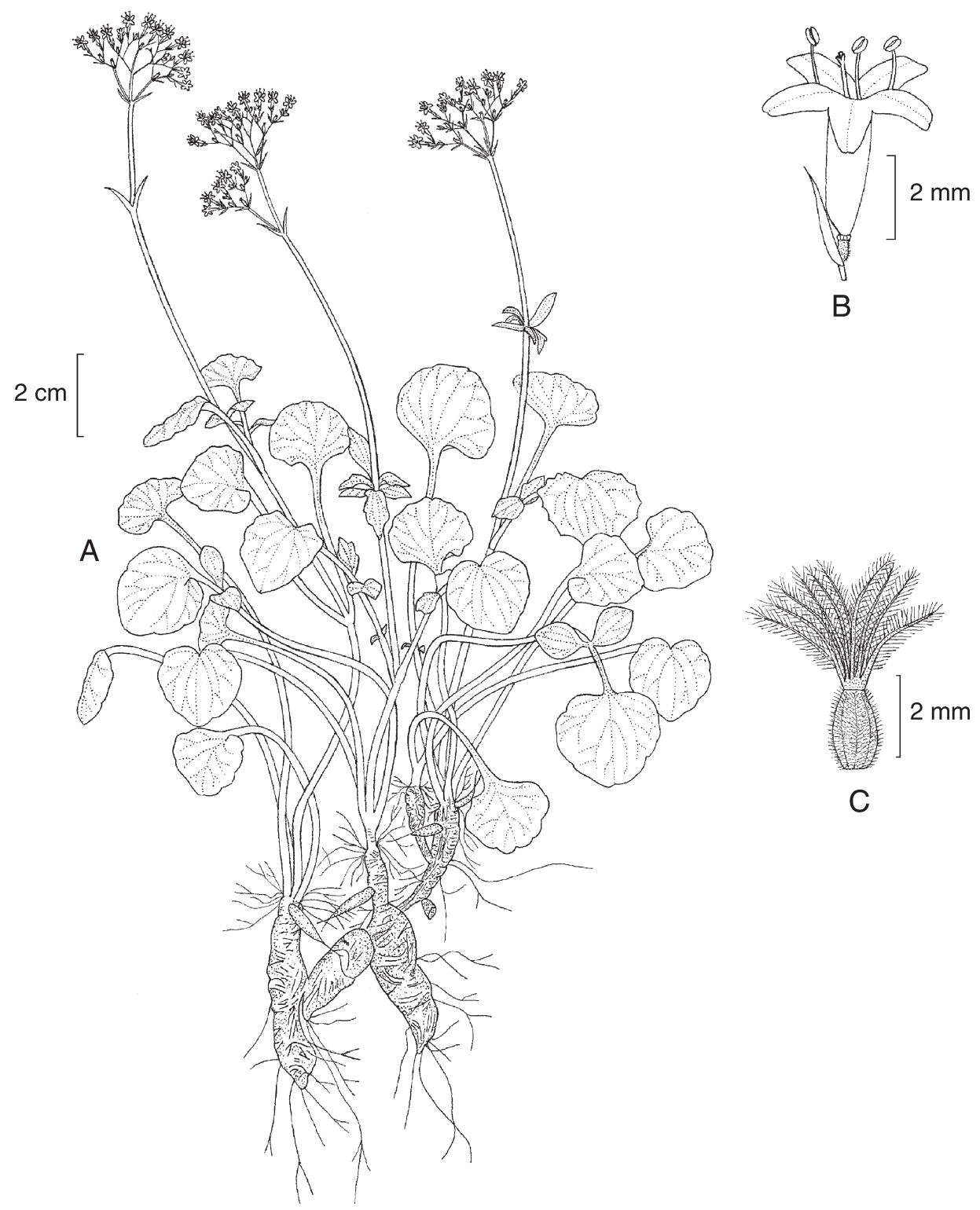

Fig. 1. Valeriana emmanuelii. A. aspecto general de la planta; B. flor; C. cara abaxial del fruto con los segmentos del cáliz expandidos. Ilustrado por Rogelio Cárdenas. 
largamente exserto; fruto ovado a piriforme, de ca. $2.2 \mathrm{~mm}$ de largo y $1.1 \mathrm{~mm}$ de ancho, de color verdoso con manchas cafés, densamente pubérulo en ambas caras, cara adaxial con una vena central simple, cara abaxial con 3 venas simples, las laterales paralelas a los márgenes.

Tipo: México, Michoacán: parte alta del cerro Campanario, $4 \mathrm{~km}$ al SE de Agua Zarca, municipio de Morelia, afloramientos peñascosos en medio del bosque de pino y encino, alt. 2300 m, 27.VII.2002, J. Rzedowski 53966 (holotipo IEB, isotipos por distribuirse).

Material adicional examinado: México, Michoacán: parte alta del Cerro Campanario, municipio de Morelia, E. Pérez y S. Zamudio 3648 (IEB); 1 km al W de Agua Zarca, próxima a San Miguel del Monte, municipio de Morelia, J. Rzedowski 53974 (IEB).

Valeriana emmanuelii se conoce solamente de dos localidades, ambas situadas en el extremo sur del municpio de Morelia, donde la planta se ha colectado en el ambiente general de bosque de pino y encino, de preferencia en afloramientos peñascosos de roca ígnea, entre 2200 y $2350 \mathrm{~m}$ de altitud. Se ha encontrado en flor en julio y en agosto.

En ambos sitios el taxon se ha visto representado en forma escasa, más bien esporádica. Todos los individuos encontrados son hermafroditas, pero dado su reducido número no cabe excluir la posibilidad de que se pueda tratar de una especie ginodioica.

Valeriana emmanuelii podría ubicarse en la serie Densiflorae definida por Meyer (1951), misma que no está sancionada formalmente por Barrie (1990). Este último autor, por otra parte, reconoce el "grupo de $V$. densiflora" con seis especies, a grandes rasgos caracterizado por plantas perennes de raíces no muy voluminosas, hojas imparipinnadas con foliolos indivisos, o bien, algunas simples, corola glabra por fuera y pilosa en la garganta por dentro, estambres y estilo exsertos, todos sus componentes distribuidos en México.

El nuevo taxon pertenece también a este grupo y podría estar relacionado con $V$. retrorsa Fern., registrada de Morelos y Estado de México, con la que comparte el carácter de la reproducción vegetativa mediante raíces gemíferas. V. retrorsa se distingue, sin embargo, en la pubescencia retrorsa de sus tallos y peciolos, en las hojas caulinas con mayor número de foliolos, mismos que suelen ser notablemente más angostos y en las corolas más largas de sus flores hermafroditas. Para la mencionada especie se registran también individuos femeninos, al parecer ausentes en $V$. emmanuelii.

A su vez, Valeriana densiflora Benth., conocida del este de Michoacán al Distrito Federal, se asemeja más a $V$. emmanuelii en la forma de sus hojas, pero difiere en su falta de follaje basal y de raíces gemíferas, así como en sus flores hermafroditas más grandes y frecuentemente rosadas, al igual que en la presencia de plantas femeninas.

El nombre de la especie está dedicado al M. en C. Emmanuel Pérez Calix, esforzado y valioso compañero de trabajo, quien fue el primero en localizar esta especie de Valeriana en los riscos del Cerro Campanario. 
Rzedowski y G. Calderón de Rzedowski: Dos Especies Nuevas de Valeriana de México

Valeriana zamoranensis Rzedowski \& Calderón sp. n.

Herba perennis ut videtur hermaphrodita usque ad $50 \mathrm{~cm}$ alta; radix manifeste incrassata ut videtur napiformis plus quam $6 \mathrm{~cm}$ longa et lata; caules 1 vel 2, in lineis longitudinalibus puberuli; folia in rosula basali aggregata, petioli ad $3.5 \mathrm{~cm}$ longi, laminae ambitu ellipticae vel obovatae 5-18 longae et 2-10 cm latae, profunde et irregulariter bitripinnatisectae segmentis linearibus, oblongis vel triangularibus, saepe falcatis, $0.5-3 \mathrm{~mm}$ latis; inflorescentia dichasiali-paniculata laxa $15-28 \mathrm{~cm}$ longa et $5-15 \mathrm{~cm}$ lata, ramuli terminales 4-7 floribus in cincinnis dispositis; corolla infundibuliformis ca. $4 \mathrm{~mm}$ longa, extus glabra, intus ad faucem pilosa; stamina stylusque exserta; fructus pyriformis ad $4 \mathrm{~mm}$ longus et $2 \mathrm{~mm}$ latus glaber, pagina abaxialis 3-nervata, 2 nervos laterales incrassatos ad margines parallelos ferens.

Planta herbácea perenne, al parecer hermafrodita, de alrededor de $50 \mathrm{~cm}$ de alto; raíz manifiestamente engrosada, en apariencia napiforme y bifurcada, de unos $6 \mathrm{~cm}$ de largo y de diámetro; tallos 1 ó 2 partiendo de la base, pubérulos en bandas longitudinales con los pelos retrorsos, más escasos a casi ausentes hacia la inflorescencia, pero condensándose a nivel de los nudos; hojas concentradas en forma de roseta basal, peciolo hasta de $3.5 \mathrm{~cm}$ de largo, envainante, a menudo ciliado, lámina elíptica a obovada en contorno general, de 5 a $18 \mathrm{~cm}$ de largo y 2 a $10 \mathrm{~cm}$ de ancho, profundamente bipinnati- a tripinnatipartida, las divisiones principales 3 a 7, opuestas o alternas, hasta de $6 \mathrm{~cm}$ de largo, irregular y delicadamente divididas en segmentos lineares, oblongos o triangulares de 0.5 a $3 \mathrm{~mm}$ de ancho, con frecuencia falcados, glabra o casi glabra, de textura membranácea; inflorescencia terminal, en forma de panícula dicasial laxa, de 15 a $28 \mathrm{~cm}$ de largo y 5 a $15 \mathrm{~cm}$ de ancho, con las ramas ascendentes, ramillas terminales, por lo general con 4 a 7 (o más) flores densamente dispuestas en un arreglo escorpiode, brácteas de las ramificaciones principales lineares a lanceoladas, hasta de $1.5 \mathrm{~cm}$ de largo, las propias de las flores angosta a ampliamente triangulares, de 0.2 a $0.4 \mathrm{~mm}$ de largo, con los bordes hialinos y ciliados, a veces además algo pilosos, sobre todo hacia el ápice; flores al parecer todas hermafroditas; cáliz de 10 a 14 segmentos; corolas blanquecinas, en estado juvenil campanuladas, en apariencia actinomorfas, de 2 a $3 \mathrm{~mm}$ de largo, las maduras infundibuliformes, tubo de ca. $2 \mathrm{~mm}$ de largo, giboso en la base, de ca. $1.8 \mathrm{~mm}$ de diámetro, en cuyo extremo inferior se encuentra un abrupto estrechamiento a manera de cuello diminuto de ca. $0.2 \mathrm{~mm}$ de largo, lóbulos patentes o doblados hacia fuera, oblongos, de ápice redondeado o algo agudo, de 1.5 a $2 \mathrm{~mm}$ de largo y 1.2 a $1.5 \mathrm{~mm}$ de ancho, glabra por fuera, densamente pilosa en la garganta por dentro; estambres largamente exsertos, anteras de ca. $1 \mathrm{~mm}$ de largo; estilo exserto; fruto piriforme, de ca. 3.5 a $4 \mathrm{~mm}$ de largo por (1)1.5 a $2 \mathrm{~mm}$ de ancho en la base, de color amarillento a café claro, glabro, fina y densamente granuloso a papiloso sobre ambas superficies, la cara adaxial con una vena central, la abaxial además con 2 venas laterales próximas y paralelas a los márgenes, engrosadas y realzadas sobre todo hacia los extremos.

Tipo: México, Querétaro, parte alta del cerro Zamorano, municipio de Colón, zona rocosa con vegetación herbácea, alt. 3200 m, 27.VIII.1989, J. Rzedowski 48780 (IEB). 


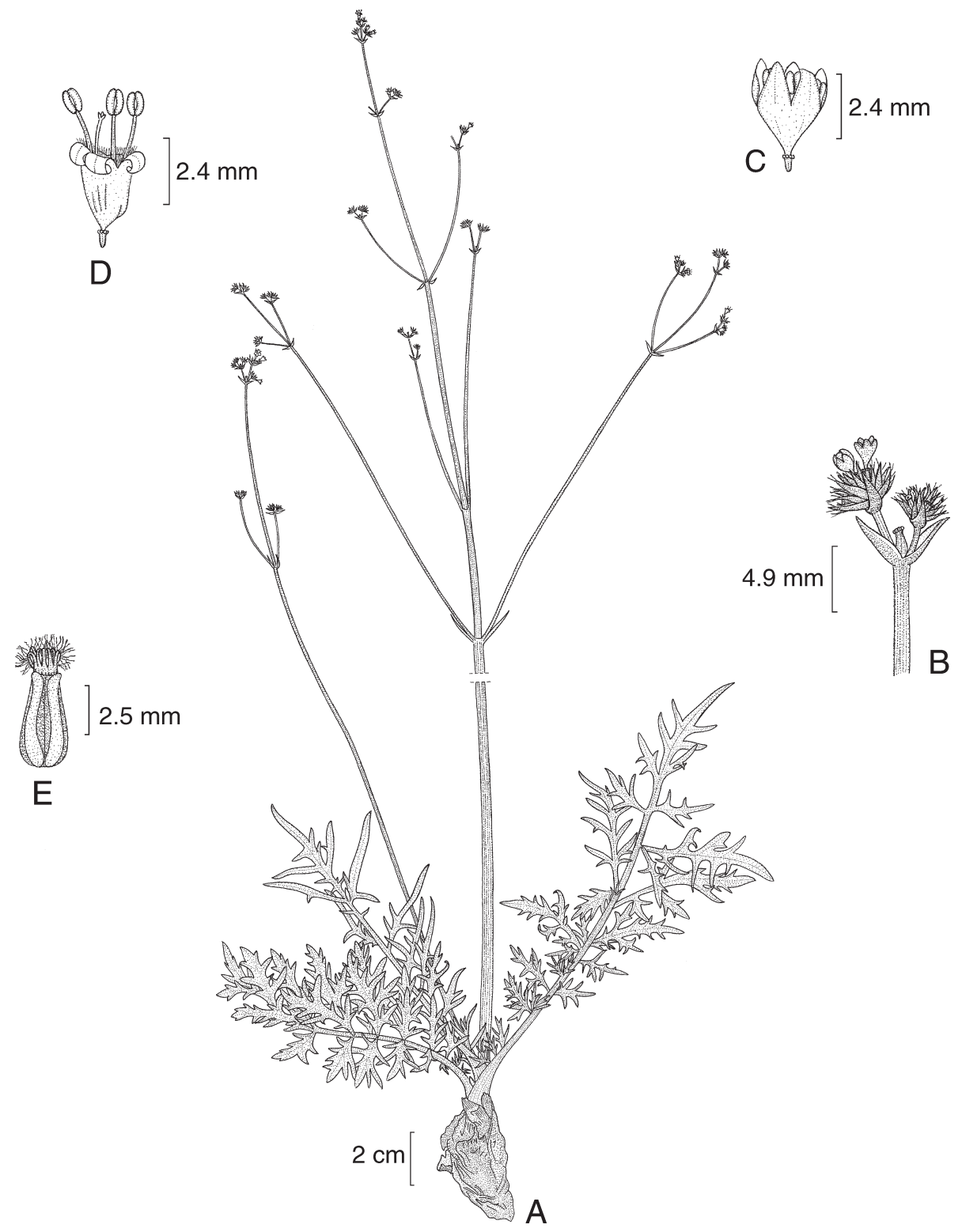

Fig. 2. Valeriana zamoranensis. A. aspecto general de la planta; B. porción de la inflorescencia; C. flor en estado juvenil; D. flor; E. cara abaxial del fruto con los segmentos del cáliz sin expanderse. llustrado por Rogelio Cárdenas. 
Valeriana zamoranensis al parecer está restringida a una pequeña área de la cumbre rocosa del Cerro Zamorano, macizo montañoso ígneo aislado, ubicado en el límite entre los estados de Querétaro y Guanajuato, donde prospera a unos $3200 \mathrm{~m}$ de altitud en medio de vegetación herbácea predominante. Son varias ya las especies de fanerógamas que se han descubierto en la parte superior de este cerro, representando endemismos muy estrechos.

Se trata de un elemento raro. Después de su colecta original realizada en 1989, la planta no ha vuelto a encontrarse, a pesar de esfuerzos especiales efectuados para localizarla. Su descripción pudo realizarse con base en dos ejemplares, uno de ellos más bien fragmentario. Aunque ambos corresponden a individuos hermafroditas, no cabe excluir la posibilidad de que la planta sea ginodioica.

V. zamoranensis debe adscribirse a la serie Ceratophyllae Hoeck. que, de acuerdo con Barrie (op. cit.), agrupa siete especies, todas distribuidas en México. Esta ubicación la indican caracteres tales como la raíz voluminosa, hojas agrupadas en roseta basal y profundamente bi- a tripinnatisectas, corolas pubescentes por dentro a nivel de la garganta y frutos con venas laterales engrosadas en la cara abaxial. En la forma de sus hojas la especie nueva se asemeja algo a V. zapotecana Barrie, conocida de Oaxaca, pero la última difiere en sus flores unisexuales y más pequeñas, así como en sus frutos pubescentes. También muestra parecido con $V$. otomiana Barrie, distribuida en San Luis Potosí, Guanajuato, Hidalgo y Querétaro, aunque la mencionada especie se diferencia en la arquitectura de la hoja, en sus flores unisexuales y en ser planta aparentemente ligada con suelos derivados de roca caliza.

\section{AGRADECIMIENTOS}

Los autores agradecen a los M. en C. Eleazar Carranza, Emmanuel Pérez Calix y al Biól. Gilberto Ocampo la ayuda prestada en la intensa búsqueda y colecta de ejemplares de Valeriana en diferentes localidades de Guanajuato, Michoacán y Querétaro. Se dan las gracias asimismo al Dr. Fred R. Barrie, quien tuvo la amabilidad de revisar los materiales de las nuevas especies y de ofrecer su valiosa opinión sobre el particular.

\section{LITERATURA CITADA}

Barrie, F. R. 1990. A systematic study of the Mexican and Central American species of Valeriana (Valerianaceae). Tesis. University of Texas. Austin, Texas. $384 \mathrm{pp}$.

Meyer, F. G. 1951. Valeriana in North America and the West Indies. Ann. Mo. Bot. Gard. 38: 377-503. 